\section{Experimentally acquired associations in memory encoding by preschoolers*}

\author{
JAMES W. HALL \\ School of Education, Northwestern University, Evanston, Ill. 60201
}

Three- and 4-year-old children were given extensive paired-associate (PA) training in which response terms were highly familiar words and stimulus terms were words semantically related to response terms but relatively unfamiliar to the Ss. Subsequent recognition memory (RM) performance indicated, by an analysis of false recognitions, that the newly acquired associations functioned during the encoding of the RM items, i.e., the PA response terms occurred as implicit associative responses to the PA stimulus terms presented for learning. The feasibility of using an entirely verbal PA task with children as young as 3 years also was demonstrated.

The present experiment examines the extent to which very young children spontaneously produce newly acquired associations during memory encoding. The general question of concern in this paper is whether the observed increase with age in the use of verbal associations during memory encoding is entirely the result of increased availability of associations or is due also to the development of some "s pontane ous production mechanism." Evidence that the occurrence of verbal associations during encoding does increase over an age range of about 7 to 12 years has been furnished by Bach \& Underwood (1970) and Felzen \& Anisfeld (1970). In these studies, the frequency of false recognitions of natural language associates of previously presented words increased with age, presumably due to an increase with experience in the strength of these associations. However, it appears possible that such differences might be due, in part at least, to a deficiency in the production of implicit associative responses (IARs) analogous to the production deficiency described by Flavell, Beach, \& Chinsky (1966), wherein very young children less frequently produced verbal mediators spontaneously during learning, although such mediators were produced on demand. Three recognition memory (RM) experiments recently conducted with 3- to 5-year-old children provided no evidence of such deficiency in the case of IAR production during learning. For example, in one of these experiments (Hall \& Halperin, 1972, Experiment II), 3-, 4-, and 5-year-olds were presented a series of highly familiar words to be remembered,

*This investigation was supported by U.S. Office of Education Grant OEG-5-71-0053(508). The author is grateful to Gay Hall, Nancy Klastorin, and Sharon Madsen for their work in data collection and to Foorman Mueller, owner and director of the Tom Thumb Nursery School. followed by a test list that included words associatively and conceptually related to certain of the to-be-remembered items. False recognitions were more frequent for the associatively and conceptually related items than for unrelated control items at every age level, and no increase with age in this respect was noted. It appeared, then, that even children as young as 3 years of age spontaneously produce IARs much as do older children and adults, provided that the relevant associations have been acquired, i.e., provided that highly familiar experimental material is employed.

A primary purpose of the present experiment was to provide further evidence concerning the question raised above by examining the extent to which 3-year-old children spontaneously produce associates encoding words for memory. A second experimental objective was a methodological one. Extensive use of standard verbal learning tasks with very young children is a relatively recent development. It is clear that such efforts are beset with difficulties having to do mainly with obtaining and maintaining the attention of Ss and posing instructions in such a way that Ss understand the tasks. We have found it necessary often to conduct special pretraining sessions, to use brief multiple sessions, and to explore various procedures through considerable pilot work. The major methodological objective of the present experiment concerned the feasibility of employing a purely verbal paired-associate (PA) task involving multiple sessions with children as young as 3 years. The idea here was first to "teach" a set of new associations to the children through PA training, then to determine, through the use of a RM task, to what extent these new associations would occur spontaneously during memory encoding. newly acquired in the laboratory when

\section{SUBJECTS}

The Ss consisted of 14 boys from 2 years, 10 months to 4 years, 1 month of age (mean $=3$ years, 7 months) and 14 girls from 2 years, 11 months to 4 years, 4 months of age (mean $=3$ years, 6 months) who were enrolled in a private nursery school in Evanston. The limited SES data available indicated that the families of these children were primarily lower middle class with some working class and upper-level professionals as well.

\section{MATERIALS AND PROCEDURE}

Phase I: PA Training

The items used in PA training are shown in Table 1. Every $S$ received the same 12 response words, each of which was judged to be highly familiar to the Ss prior to commencing PA training. For seven boys and seven girls the words in List 1a served as stimulus terms, whereas the words in List $1 \mathrm{~b}$ served as stimulus terms for the remaining Ss. As may be seen in Table 1, the stimulus terms were either subordinates of their corresponding response terms or were highly related semantically (e.g., synonyms). Since it was important that the association between pair members be relatively low at the outset of training, an oral pretest was given in which Ss were asked what each stimulus word meant. The results confirmed the desired low association, since most $\mathrm{Ss}$ indicated lack of familiarity with all of the stimulus terms.

Six of the 12 pairs were introduced in Session 1 with a study trial of the following type: "TUG. A TUG is a kind of BOAT. When I say TUG, you say BOAT." Four anticipation trials then were given, and the session was terminated. In Session 2, the remaining six pairs were introduced. The procedure in that session was the same as in Session 1, except that following the four anticipation trials on the second set of six pairs, two anticipation trials were given with the items introduced in Session 1.

The remainder of the $\mathrm{PA}$ training consisted of seven sessions of four

Table 1

Stimulus and Response Terms Used in Paired Associate Training

\begin{tabular}{lll}
\hline \multicolumn{2}{c}{ Stimuli } & \\
\cline { 1 - 2 } \multicolumn{1}{c}{ List 1a } & \multicolumn{1}{c}{ List 1b } & Responses \\
\hline BEAGLE & POODLE & DOG \\
RAPID & SPEEDY & FAST \\
POND & BEACH & LAKE \\
COTTAGE & CABIN & HOUSE \\
TACK & SPIKE & NAIL \\
HOG & PORK & PIG \\
BROOK & STREAM & RIVER \\
YACHT & TUG & BOAT \\
SLACKS & TROUSERS & PANTS \\
CHEERFUL & JOYFUL & HAPPY \\
CROW & SPARROW & BIRD \\
WOOL & LAMB & SHEEP \\
\hline
\end{tabular}


anticipation trials per session, except that (1) if a $S$ performed perfectly on two consecutive trials within a session, that session was terminated, and (2) if a $S$ performed perfectly in two consecutive sessions, the sessions were terminated. In such cases, the Ss were assigned a perfect score for those trials and/or sessions that were omitted.

The order of pairs presentation was varied randomly both within and between sessions, and the same order was used for all $S$ s receiving a particular set of pairs. Presentation rate varied somewhat according to the idiosyncracies of the Ss, but in general was such that sessions typically ran from 5 to $8 \mathrm{~min}$ in duration.

Phase II: The RM Task

The RM tasi was administered first 7-10 days following completion of the $\mathrm{PA}$ training. It was administered in two parts in order to reduce attentional problems. For each part, 10 items were read in a predetermined random order at a 4-5-sec rate, with Ss instructed to pay careful attention and to try to remember the words. After a break of about $30 \mathrm{sec}$, the test list was administered using the following format: "BOAT. Was BOAT one of the words I said to you?" The two parts were administered during a single session and were separated by a break of about $30 \mathrm{sec}$.

Among the total of 20 to-be-remembered items, randomly ordered for presentation, were 12 that had served as stimulus words ${ }^{\circ} \mathrm{O}_{i}$ ?A training. Of these 12 items, 6 were from PA List $1 \mathrm{a}$ and 6 from PA List $1 \mathrm{~b}$, i.e., only 6 had served as PA stimulus items for any given $S$. The remaining 8 to-be-remembered items also appeared in the test list and are termed repeated (R) words. In addition to these $8 \mathrm{R}$ words, the test list included the $12 \mathrm{PA}$ response words and 12 words that were unrelated to any of the to-be-remembered items (termed unrelated control or UC words). Note that for any given $\mathrm{S}$, only 6 of the $12 \mathrm{PA}$ response terms included in the RM test have been paired with any of the to-be-remembered items in the RM task. These 6 words are termed experimental associates (EA words), whereas the remaining (equally familiar) PA response terms are termed control associates (CA words), in that it is the difference in frequency of false recognitions to these two classes of words that would reveal the effects of PA training on IAR production during memory encoding.

Finally, since all Ss continued to be available for experimentation for a period of several months, the recognition memory task was again administered some 7-8 weeks following PA training to examine the persistence of effects found in the earlier testing. All items and procedures were identical to the earlier RM testing.

\section{RESULTS AND DISCUSSION PA Training}

Two Ss were eliminated from the study due to their consistent failure to follow the PA task instructions and a third because of frequent nonattendance during the period of the study. For the remaining $25 \mathrm{Ss}$, performance increased monotonicaliy across sessions for Sessions 3.9 from a mean of 24.44 (SD $=12.55)$ items correct over the four trials in a session (of a possible 48 ) to a mean of 41.72 $(\mathrm{SD}=5.67)$, or about $87 \%$, correct during the final session. These data suggest that the task was well suited to the capabilities of the Ss, none of whom had had previous experience with such a task.

It is clear from the above results that the use of a purely verbal paired-associate task with children as young as 3 years of age is entirely feasible. No previous demonstration of this feasibility was found in a search of available literature. It was necessary, of course, to modify considerably the standard labcratory procedures by introducing the full set of 12 pairs gradually and by employing a series of brief sessions rather than massing the trials in one or two longer sessions. In addition, auditory presentation was required, and the instructions given on the initial study trial were modified to stress the semantic connection between the members of pairs. Nevertheless, the task was a paired-associate one and performance did increase steadily over sessions to a relatively high performance level in the final session. Moreover, this was accomplished with Ss who had no prior associate training and who represented a rather wide range in SES and also with pairs in which stimulus items were relatively low in meaningfulness for these young children.

\section{RM Performance}

Of the 25 Ss who completed the PA training, 13 ( 6 boys and 7 girls) had received PA List $1 \mathrm{a}$ and 12 ( 6 boys and 6 girls) had received PA List $1 \mathrm{~b}$.
To insure the effectiveness of the PA counterbalancing procedure, the data for 1 of the 7 girls who received PA List 1a (randomly determined) were discarded, so that the analyses of the RM data are based on 24 Ss, 6 in each of the counterbalancing conditions.

In the first RM session, the mean percentages of judgments of "old" were 84 for $R$ words, 58 for $A E$ words, 38 for $\mathrm{AC}$ words, and 34 for UC words. Major interest was in a comparison of $\mathrm{AE}$ and $\mathrm{AC}$ words in frequency of false recognitions (i.e., judgments of "old"). That mean difference of $1.16(\mathrm{SD}=1.81)$ was highly significant $[\mathrm{t}(23)=3.09$, $\mathrm{p}<.01]$. Note that the $\mathrm{AC}$ and $\mathrm{UC}$ items differed only slightly and noisignificantly in that respect.

In the second RM session, the mean percentages of "old" judgments were 82 for $\mathrm{R}$ words, 33 for $\mathrm{AE}$ words, 21 for $\mathrm{AC}$ words, and 13 for $\mathrm{UC}$ words. The mean difference between $\mathrm{AE}$ and $\mathrm{AC}$ words of $.67(\mathrm{SD}=1.72)$ was of borderline reliability $[\mathrm{t}(23)=1.85$, $.10>p>.05$. Again, the difference between the $\mathrm{AC}$ and $\mathrm{UC}$ items was not significant.

These data indicate that after 1 week and probably also 7-8 weeks following PA training, the newly acquired associations functioned during the encoding of the RM items. Such results offer no support for the notion of an IAR production deficiency as introduced at the outset of this paper. However, the present data do not preclude such a possibility. That is, it is possible that the acquisition of new associations by older children would have resulted in even greater use of them for memory encoding. In any case, it appears that newly acquired verbal associations are readily activated during memory encoding by children as young as 3 years.

\section{REFERENCES}

BACH, M. J., \& UNDERWOOD, B. J. Developmental changes in memory attributes Journal of Educational Psychology, 1970,61, 292-296.

FELZEN, E., \& ANISFELD, M. Semantic and phonetic relations in the false recognition of words by third- and sixth-grade children. Developmental Psychology, 1970,3, 163-168.

FLAVELL, J. H., BEACH, D. H., \& CHINSKY, J. M. Spontaneous verbal rehearsal in a memory task as a function of age. Child Development, 1966, 37, 283-299.

HALL, $W$ \& HALPERIN, M. S. The development of memory-encoding processes in young children. Developmental Psychology, 1972, 6, 181. 